\title{
Effects of ATX-MS-1467 immunotherapy over 16 weeks in relapsing multiple sclerosis
}

Jeremy Chataway, MA, PhD, FRCP, Keith Martin, PhD, MRPharmS, Kevin Barrell, PhD, Basil Sharrack, MD, PhD, FRCP, Pelle Stolt, PhD, and David C. Wraith, PhD, For the ATX-MS1467 Study Group

Neurology ${ }^{\circledR}$ 2018;90:e955-e962. doi:10.1212/WNL.0000000000005118

\section{Abstract}

\section{Objective}

To assess safety, tolerability, and efficacy of the antigen-specific immunotherapy ATX-MS-1467 in participants with relapsing multiple sclerosis using different treatment protocols to induce tolerance.

\section{Methods}

Two open-label trials in adult participants with relapsing multiple sclerosis were conducted. Study 1 was a multicenter, phase 1 b safety evaluation comparing intradermal (i.d.) (cohort 1) with subcutaneous (cohort 2) administration in 43 participants. Both cohorts received ATXMS-1467 dosed at 25, 50, 100, 400, and $800 \mu \mathrm{g}$ at 14-day intervals over 8 weeks, followed by 8 weeks with 4 additional 800 - $\mu$ g doses at 14-day intervals and 32 weeks off study medication. Study 2 was a phase 2 a, multicenter, single-arm trial enrolling 37 participants. ATX-MS-1467 was titrated from $50 \mu \mathrm{g}$ i.d. on day 1 to $200 \mu \mathrm{g}$ on day 15 and $800 \mu \mathrm{g}$ on day 29 followed by biweekly administration of $800 \mu \mathrm{g}$ for 16 weeks and 16 weeks off study medication. Efficacy was evaluated on MRI parameters and clinical variables. Safety endpoints included treatmentemergent adverse events and injection-site reactions.

\section{Results}

In study 1 , there was a significant decrease in new/persisting $\mathrm{T} 1$ gadolinium-enhanced (GdE) lesions in cohort 1 from baseline to week 16, returning to baseline values at week 48 . In study 2 , the number of $\mathrm{T} 1 \mathrm{GdE}$ lesions were significantly reduced on treatment and remained reduced at study completion. Safety results were unremarkable in both studies.

\section{Conclusion}

Relatively slow ATX-MS-1467 titration and a longer full-dose i.d. treatment period is associated with reduction in GdE lesions and a sustained effect post treatment. Further trials of ATX-MS1467 are warranted.

\section{Classification of evidence}

This work provides Class IV evidence that for patients with relapsing multiple sclerosis, slow ATX-MS-1467 titration and a longer full-dose i.d. treatment period is associated with reduction in GdE lesions.

\author{
Correspondence \\ Dr. Chataway \\ j.chataway@ucl.ac.uk
}

MORE ONLINE

$\rightarrow$ Class of Evidence

NPub.org/coe

From the Queen Square Multiple Sclerosis Centre (J.C.), Department of Neuroinflammation, UCL Institute of Neurology, University College London; Apitope Technology (Bristol) Ltd. (K.M., K.B., D.W.), Chepstow; Academic Department of Neuroscience (B.S.), NIHR Sheffield Neuroscience Biomedical Research Centre, University of Sheffield, UK; MagliaRotta (P.S.), Basel, Switzerland; and Institute of Immunology and Immunotherapy (D.C.W.), Birmingham, UK. 


\section{Glossary}

$\mathbf{A E}=$ adverse event $; \mathbf{A R R}=$ annualized relapse rate $;$ EDSS $=$ Expanded Disability Status Scale; GdE = gadolinium-enhanced; i.d. $=$ intradermal; ITT $=$ intention-to-treat; $\mathbf{M B P}=$ myelin basic protein; $\mathbf{m I T T}=$ modified intention-to-treat; $\mathbf{M S}=$ multiple sclerosis; MSFC = Multiple Sclerosis Functional Composite; RMS = relapsing multiple sclerosis; s.c. $=$ subcutaneous; TEAE = treatment-emergent adverse event.

By aiming to reinstate tolerance to the protein causing the disease, ${ }^{1}$ antigen-specific immunotherapy represents a major conceptual shift away from most current and emerging immunomodulatory therapies for multiple sclerosis (MS). ${ }^{2-7}$

Peripheral tolerance is mediated by steady-state dendritic cells when peptides bind to major histocompatibility complex class II molecules at their surface, promoting interaction with antigen-specific $\mathrm{T}$ cells and subsequent generation of regulatory $\mathrm{CD} 4^{+} \mathrm{T}$ cells. ${ }^{8}$ The risk of an immune response can be avoided during tolerance induction by the use of synthetic peptides that mimic naturally processed $\mathrm{CD}^{+} \mathrm{T}$ cell epitopes. ${ }^{9,10}$ Such peptides have been termed apitopes, short for antigen-processing independent epitopes. ${ }^{11}$

We have identified epitopes from 4 regions of myelin basic protein (MBP) that behave as apitopes but also induce tolerance to myelin and reduce disease severity by up to $95 \%$ in a humanized mouse model when administered subcutaneously (s.c.) as a cocktail (ATX-MS-1467). ${ }^{11}$ In an initial phase 1 trial of 6 participants with secondary progressive MS, ATX-MS-1467 was well tolerated, and there were significant immunologic changes consistent with a clinical effect on $\mathrm{T}$ cell responses to MBP 1 month after treatment compared to visit $1 .^{11}$

The most appropriate ATX-MS-1467 titration scheme still needs to be identified. Accordingly, we performed 2 follow-up studies in participants with relapsing MS (RMS): one phase $1 \mathrm{~b}$ ascending-dose safety evaluation comparing intradermal (i.d.) with s.c. administration and one proof-of-concept phase 2a trial assessing the effects on MRI parameters of ATX-MS1467 administered i.d. and using a shorter dose-escalation phase with a prolonged highest-dose period.

\section{Methods}

\section{Primary research questions}

The aim of the 2 studies was to assess safety, tolerability, and efficacy of ATX-MS-1467 in RMS using different treatment protocols to induce tolerance.

\section{Standard protocol approvals, registrations, and patient consents}

Both studies were entered into public registries: the phase $1 b$ study in the European Clinical Trials Database (number 2009-016710-25) and the phase $2 \mathrm{a}$ study on the ClinicalTrials.gov registry (identifier: NCT01973491). The studies were conducted in accordance with all applicable regulations, including the International Conference on Harmonisation Good Clinical Practice Guidelines and the ethical principles in the Declaration of Helsinki, ${ }^{12}$ as well as local regulations and standards. The appropriate institutional review boards or regional review boards approved the use of human subjects for the studies. All participants gave written informed consent.

\section{Participants}

The studies were open-label trials in adult participants aged 18 to 55 years (study 1 ) and 18 to 65 years (study 2), respectively, with RMS ( $\geq 1$ documented relapse in the previous 12 months or 2 relapses within the previous 24 months), as defined by the McDonald 2010 criteria, $^{13}$ positive for human lymphocyte antigen (HLA)-DRB1*15, and with Expanded Disability Status Scale (EDSS) scores $\leq 5.5$. Prior and concomitant disease-modifying therapies were prohibited, except for interferon beta where adequate washout was required prior to study entry. Corticosteroids were permitted to treat relapses.

\section{Phase 1 b study (study 1)}

The phase $1 \mathrm{~b}$ study was a multicenter, 2 -arm, ascending-dose safety and proof-of-principle study with the primary objective to assess the safety of ATX-MS-1467. Secondary objectives were to assess, on an exploratory level, the effect of ATX-MS1467 on brain MRI (gadolinium-enhanced [GdE], 1.5-tesla MRI scanning).

The study enrolled 43 participants at 9 centers in the Russian Federation and 2 centers in the United Kingdom. Participants were divided into 2 cohorts. ATX-MS-1467 was administered i.d. in cohort 1 and s.c. in cohort 2. Participants were allocated to a cohort consecutively in the order of enrollment into the study. Dosing, titration, and intervals (figure 1A) were based on the earlier phase 1 study. ${ }^{11}$ Over an 8 -week titration period, both cohorts received increasing doses of ATX-MS-1467 of $25,50,100,400$, and $800 \mu \mathrm{g}$ at $14 \pm 3$ day intervals. The titration period was followed by an 8 -week, full-dose period during which participants received 4 additional $800-\mu \mathrm{g}$ doses at $14 \pm 3$ day intervals. Participants were then followed for an additional 32 weeks off study medication.

Safety was assessed by the incidence of adverse events (AEs) and treatment-emergent AEs (TEAEs), examination of safety laboratory values, anti-peptide antibody tests, urine pregnancy tests, vital signs, physical examination, neurologic examination, and MRI evaluation of brain lesions. A TEAE was 
Figure 1 Designs of the 2 studies

\begin{tabular}{|c|c|c|c|}
\hline $\begin{array}{cc}\text { Screening } & \text { Predose } \\
\text { period } & \text { period } \\
\text { (4 weeks) } & \text { (4 weeks) }\end{array}$ & $\begin{array}{l}\text { Titration } \\
\text { period } \\
\text { (8 weeks) }\end{array}$ & $\begin{array}{c}\text { Full-dose treatment } \\
\text { period } \\
\text { (8 weeks) }\end{array}$ & $\begin{array}{l}\text { Off-treatment } \\
\text { follow-up } \\
\text { (32 weeks) }\end{array}$ \\
\hline Off treatment & $\begin{array}{c}\text { ATX-MS-1467 } \\
25,50,100,400,800 \\
\mu g \text { (s.c./i.d. cohorts } \\
\text { 1/2) biweekly }\end{array}$ & $\begin{array}{l}\text { ATX-MS-1467 } \\
800 \mu \text { (s.c./i.d. } \\
\text { cohorts } 1 / 2 \text { ) } \\
\text { biweekly }\end{array}$ & Safety and efficacy evaluation \\
\hline
\end{tabular}
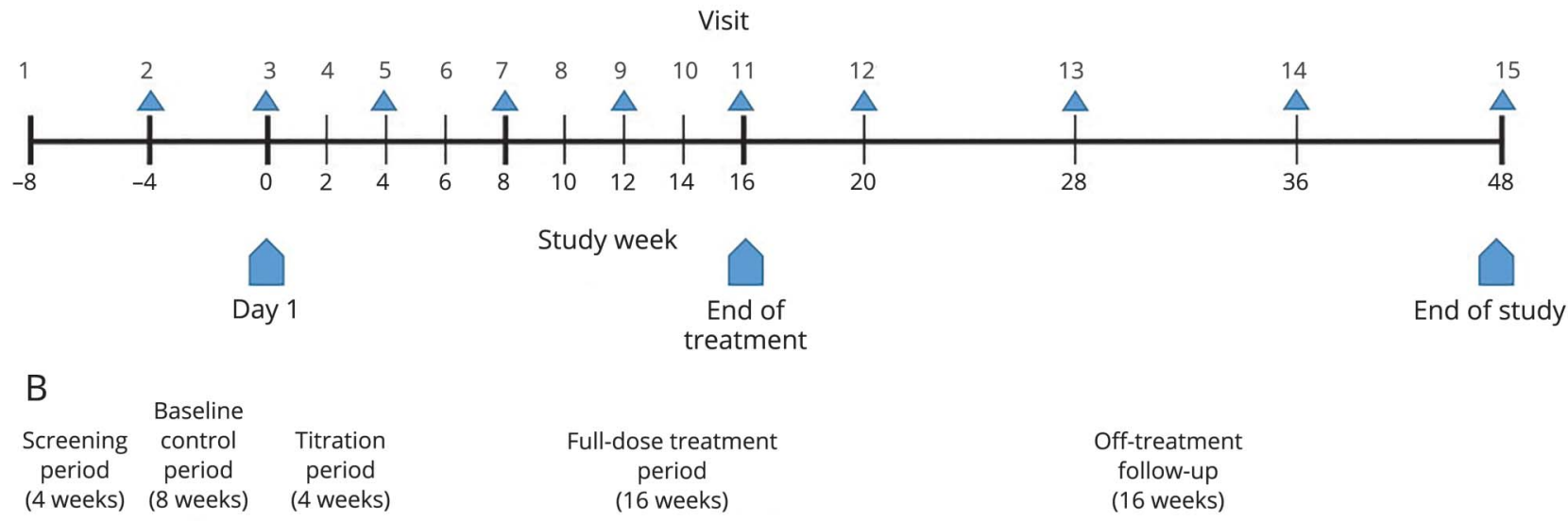

treatment

\begin{tabular}{|c|c|c|c|}
\hline Off treatment & $\begin{array}{c}\text { ATX-MS-1467 } \\
50,200,800 \mu \mathrm{g} \\
\text { i.d. biweekly }\end{array}$ & $\begin{array}{c}\text { ATX-MS-1467 } \\
800 \mu \mathrm{g} \text { i.d. biweekly }\end{array}$ & Evaluation of maintenance of treatment effect \\
\hline
\end{tabular}

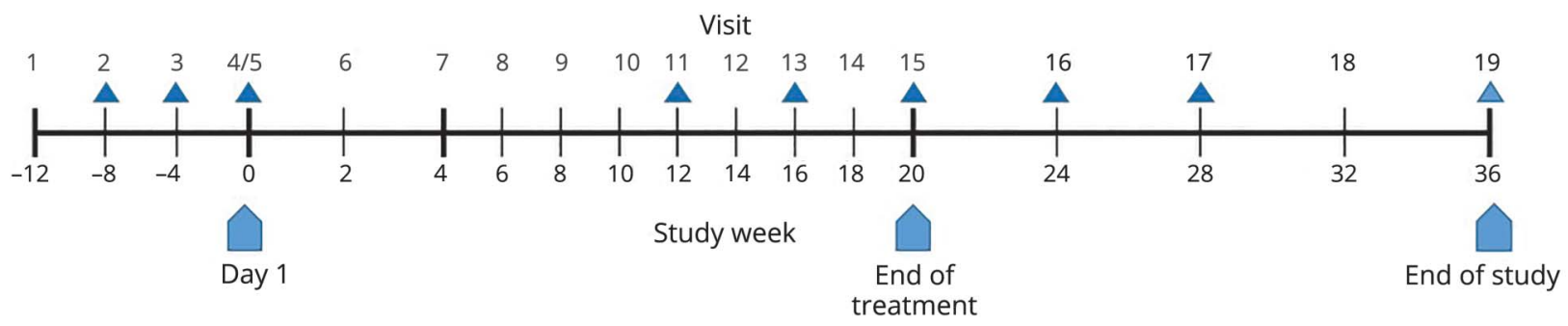

Time course, titration schemes, and assessment points are shown for the 2 studies. (A) Study 1 (phase $1 \mathrm{~b}$ study); (B) study 2 (phase 2 a study). Triangles indicate MRI measurements. i.d. = intradermal; s.c. = subcutaneous.

defined as an AE occurring on or after the first administration of ATX-MS-1467 and no later than week 20. A data monitoring committee reviewed the combined safety data for cohort 1 after the last dose of $800 \mu \mathrm{g}$ was administered to the first 10 and 20 participants, respectively. The primary safety endpoints were evaluated on an ongoing basis up to week 20 and again at week 48. Safety was analyzed in the intention-totreat (ITT) population, defined as all participants who received $\geq 1$ administration of ATX-MS-1467 any time during the study.

Efficacy was assessed based on MRI evaluation of brain lesions as part of the safety evaluation on a proof-of-principle basis. Analyses included the presence of new T1 GdE lesions, new or enlarged T2 hyperintense lesions, and T1 hypointense lesions. The analyses were performed on the MRI population, defined as the per-protocol population (all ITT participants who complied with the protocol up to and including the week20 visit).

In this explorative study, no formal sample size calculation was performed, and $p$ values are presented for descriptive purposes only.

In both studies, continuous variables are summarized descriptively using the number of observations, means $\pm \mathrm{SD}$, 95\% confidence interval, median, minimum, and maximum. Categorical variables are summarized using frequency counts and percentages.

\section{Phase 2a study (study 2)}

The phase 2a study was a multicenter, single-arm, proof-ofconcept trial at 7 sites in the Russian Federation and 1 site in Latvia. The primary objective was to evaluate the effects of 
ATX-MS-1467 administered i.d. compared with a baseline control period off treatment. Safety evaluation was a secondary objective.

The dosing, escalation, and intervals differed from the phase 1 studies in that a shorter titration regimen was used, followed by a longer highest-dose treatment period (figure 1B). During a 4-week titration period, the dose of ATX-MS-1467 was titrated from $50 \mu \mathrm{g}$ on day 1 to $200 \mu \mathrm{g}$ on day 15 and $800 \mu \mathrm{g}$ on day 29. Subsequently, participants received biweekly administration of $800 \mu \mathrm{g}$ ATX-MS-1467 for 16 weeks. Participants were then followed for an additional 16 weeks off study medication.

Efficacy was evaluated on MRI parameters and clinical variables. The primary endpoint was change in the number of $\mathrm{T} 1$ GdE lesions at the last 3 on-treatment scans compared with the number of T1 GdE lesions at the 3 baseline scans. Secondary endpoints included number and change from baseline in T1 GdE lesions at each visit post baseline, the number of new or newly enlarging T2 lesions post baseline, mean annualized relapse rate (ARR) at week 20, time to first relapse, and change from baseline in total EDSS scores and in total Multiple Sclerosis Functional Composite (MSFC) scores, both assessed at week 20. Relapses were objectively assessed by the investigator.

Safety endpoints included TEAEs, injection-site reactions, vital signs, clinical laboratory variables, ECGs, and the frequency and timing of premature study termination. A TEAE was defined as any AE occurring on or after the first dose and within 28 days after the date of last dose.

The sample size calculation assumed a $70 \%$ reduction in GdE lesions with treatment compared with baseline, with $5 \pm 6$ $\mathrm{GdE}$ lesions on average at baseline and 1.5 $\pm 1.8 \mathrm{GdE}$ lesions on average during the posttreatment period. ${ }^{14}$ Using a 2 -sided $5 \%$ level, $>80 \%$ and $>90 \%$ power was reached with sample sizes of 12 and 14 participants, respectively. Thus, a sample size of 15 participants was selected.

Primary and secondary endpoints were analyzed in the modified ITT (mITT) population, which included all enrolled participants who received $\geq 1$ dose of ATX-MS-1467 and had $\geq 2$ MRI scans during the baseline control period and $\geq 2$ MRI scans during planned on-treatment visits. Maintenance of response was analyzed in the "responders" population (participants in whom there was a $\geq 60 \%$ reduction from baseline in the number of T1 GdE lesions at week 20), and safety analyses were based on the safety population.

The primary endpoint was analyzed using a nonparametric Wilcoxon signed rank test. A supportive analysis was performed to estimate mean percentage reduction from baseline in new T1 GdE lesions using a generalized estimating equations linear regression model with negative binomial and Poisson link functions. Descriptive statistics were used for secondary endpoints at each applicable visit for the mITT analysis set. Time to event variables are presented as Kaplan-Meier estimates, median survival, and 95\% confidence interval.

\section{Results}

\section{Study populations}

Flow diagrams for the 2 studies are shown in figure 2. In study 1 , the ITT population consisted of 43 participants, the perprotocol population of 35 , and the MRI population of 37 participants. A total of 42 participants completed the treatment phase of the study: 20 in cohort 1 and 22 in cohort 2 . Nineteen participants in cohort 1 and 20 participants in cohort 2 completed the follow-up period; 2 participants discontinued and 1 was lost to follow-up. One participant attended all follow-up visits but did not receive the last dose of ATX-MS-1467 because of concerns of an allergic reaction and was not included in the follow-up analysis population.

In study 2, 37 participants were enrolled, of whom 19 completed the titration period and were included in the ITT, mITT, and safety populations. One participant discontinued before the end of the treatment period (because of diarrhea, recorded as an $\mathrm{AE}$ ) and one participant withdrew consent after completing the treatment period. Seven participants demonstrated a $\geq 60 \%$ reduction from baseline in the number of T1 GdE lesions at week 20 and were included in the responder population.

Demographic characteristics at baseline are shown in the table. In both studies, all participants were Caucasian with mean ages approximately 30 years and 70\% female. EDSS scores and number of MS relapses in the last 24 months were similar in both studies. The study 1 population had fewer GdE lesions at baseline $(2.8 \pm 6.80)$ than the study 2 population $(7.4 \pm 7.62)$.

\section{Efficacy results}

In study 1 , there was a significant $73 \%$ decrease in the number of new or persisting GdE lesions in cohort 1 from $3.4 \pm 8.23$ at baseline (week 0$)$ to $0.9 \pm 1.67$ at week $16(p=0.030$; figure $3 \mathrm{~A})$. The number of new or persisting lesions had returned to baseline values by study end. In cohort 2 , the mean number of new or persisting $\mathrm{GdE}$ lesions was relatively low throughout the study, increasing moderately to week 16 but returning to close to baseline values by week 48 . The values were considered to represent normal variation.

The total volume of GdE lesions in cohort 1 decreased from $0.305 \mathrm{~mL}$ at study day 1 to $0.137 \mathrm{~mL}$ at week 16 (a $0.187 \mathrm{~mL}$ decrease) and remained reduced at week 48 . The lowest value $(0.094 \mathrm{~mL})$ was measured at week 36 (figure 3B). In cohort 2 , there was greater variability throughout, and the lesion volume at week 48 was comparable to that at baseline.

At week 48, 70.6\% (12/17) of participants in cohort 1 were free of GdE lesions, an increase from $52.9 \%(9 / 17)$ at 


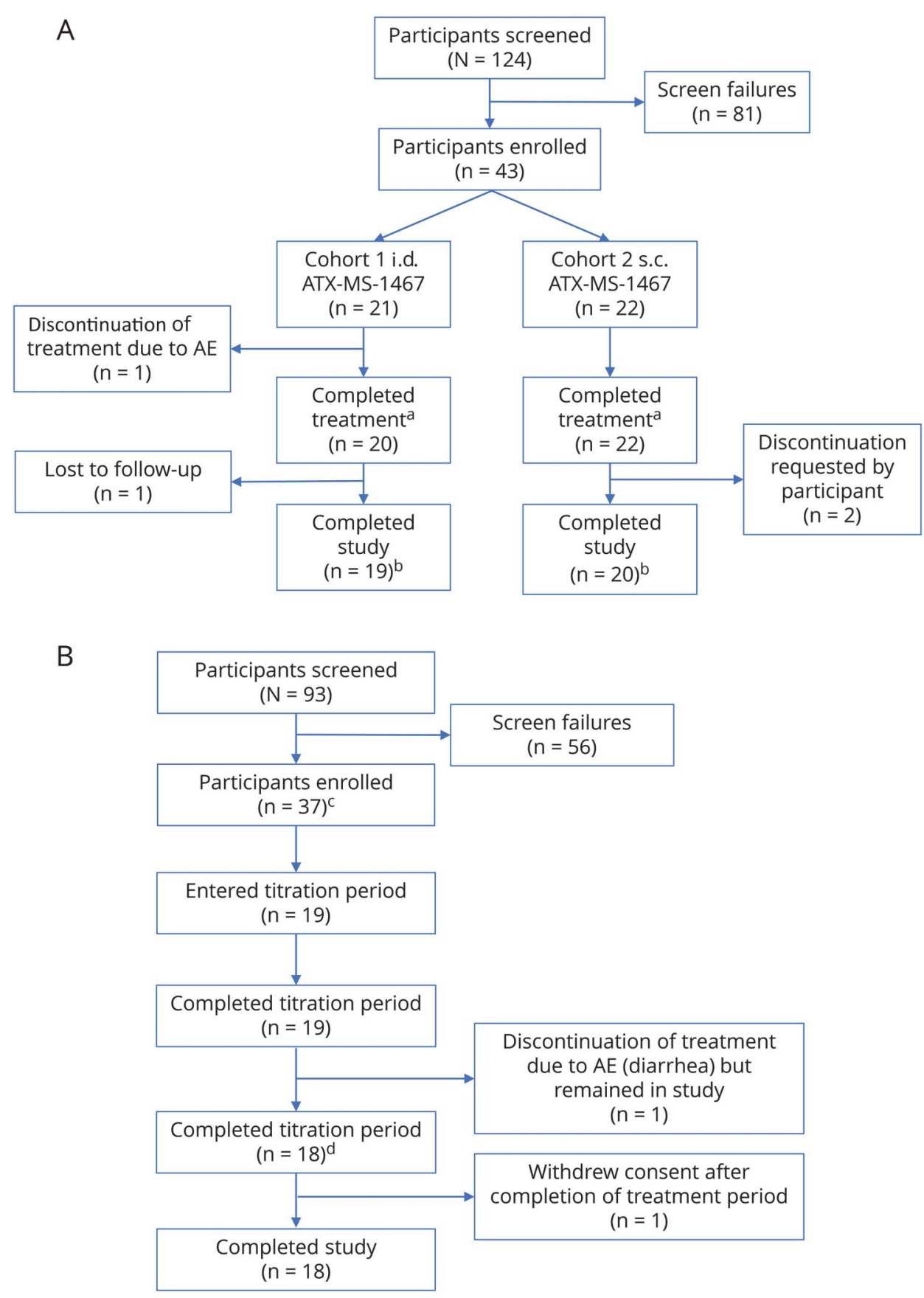

baseline. In cohort 2, the percentages of participants free of GdE lesions were comparable at baseline and week 48. There were no notable changes in the mean number of new or newly enlarging $\mathrm{T} 2$ hyperintense lesions.

In study 2 , there was a statistically significant decrease in the number of T1 GdE lesions on treatment (average of up to 3 measurements at weeks 12,16, and 20) compared with baseline (from $7.4 \pm 7.62$ to $5.0 \pm 7.24$; $p=0.0143$ ) based on a nonparametric analysis. Number and volume of T1 GdE lesions remained reduced from the end of the treatment period to the study end (figure 4). The mean reduction in lesions from baseline ranged from 1.6 to 4.6 , and the reduction in lesion volume ranged from 0.579 to $0.225 \mathrm{~mL}$.

Changes from baseline to the end of treatment in EDSS and MSFC scores were not significant. There were numerical improvements ( $p=0.054$, Wilcoxon matched-pairs signed rank test) during the study in the MSFC score from baseline to the end of the treatment period. This observation drove a post hoc analysis on the MSFC individual score components. The Paced Auditory Serial Addition Test score 
Table Demographic characteristics of the study populations at baseline

\begin{tabular}{|c|c|c|c|}
\hline \multirow[b]{2}{*}{ Variable } & \multicolumn{2}{|c|}{ Study $1(n=43)^{a}$} & \multirow[b]{2}{*}{ Study $2(n=19)^{b}$} \\
\hline & Cohort 1 & Cohort 2 & \\
\hline Age, y & $33.0 \pm 9.5$ & $31.6 \pm 9.6$ & $27.1 \pm 5.5$ \\
\hline Sex, female & $17(81)$ & $13(59)$ & $15(79)$ \\
\hline Height, cm & $164.9 \pm 7.9$ & $168.9 \pm 8.1$ & $168.8 \pm 6.9$ \\
\hline Weight, kg & $66.2 \pm 15.6$ & $67.8 \pm 12.8$ & $65.49 \pm 16.7$ \\
\hline BMI, $\mathrm{kg} / \mathrm{m}^{2}$ & $24.24 \pm 4.9$ & $23.70 \pm 3.6$ & $22.78 \pm 4.4$ \\
\hline EDSS score & $2.52 \pm 0.9$ & $2.05 \pm 0.8$ & $2.32 \pm 0.8$ \\
\hline MSFC score & NA & NA & $0.289 \pm 0.52$ \\
\hline No. of T1 GdE lesions ${ }^{c}$ & $3.4 \pm 8.2$ & $2.3 \pm 5.5$ & $7.4 \pm 7.6$ \\
\hline $\begin{array}{l}\text { Volume of T1 } \\
\text { GdE lesions, } \mathrm{mL}^{\mathrm{c}}\end{array}$ & $0.305 \pm 0.61$ & $0.189 \pm 0.43$ & $0.838 \pm 1.02$ \\
\hline \multicolumn{4}{|l|}{$\begin{array}{l}\text { MS relapses in } \\
\text { previous } 24 \text { mo }\end{array}$} \\
\hline $1-2$ & $18(86)$ & $22(100)$ & $17(90)$ \\
\hline $3-4$ & $3(14)$ & 0 & $2(10)$ \\
\hline ARR & & $0.58-1.11^{d}$ & \\
\hline $\begin{array}{l}\text { Time since last } \\
\text { MS relapse, mo }\end{array}$ & NA & NA & $4.2 \pm 3.3$ \\
\hline
\end{tabular}

Abbreviations: $\mathrm{ARR}=$ annualized relapse rate; $\mathrm{BMI}=$ body mass index; EDSS = Expanded Disability Status Scale; GdE = gadolinium-enhanced; MS = multiple sclerosis; MSFC = Multiple Sclerosis Functional Composite; NA = not assessed.

Data represent either mean \pm SD or $n(\%)$.

${ }^{a}$ Intention-to-treat population.

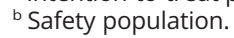

c MRI population; $\mathrm{n}=37$

${ }^{d}$ ARR was estimated from the numbers of recorded relapses in the previous 24 months. The lowest value in the range assumes that all patients had 1 or 3 relapses, respectively; the high range assumes 2 or 4 relapses.

improved significantly $(p=0.01)$ from week 0 to the end of the treatment period.

During study treatment, 3 participants (15.8\%) experienced single relapses (on days 50,59, and 89, respectively) corresponding to an ARR of 2.60 for these patients ( 0.41 for the study population). Participants received rescue corticosteroid therapy. $A \geq 85 \%$ treatment effect was maintained between week 20 and study end for $57 \%$ of those participants (7) of the study population who showed $>60 \%$ decrease in new $\mathrm{T} 1$ lesions.

\section{Safety and tolerability}

In study 1, 33 participants (77\%) experienced a TEAE, all of which were mild or moderate. Twenty participants (47\%) experienced treatment-related TEAEs, 14 in cohort 1 (67\%) and $6(27 \%)$ in cohort 2 . The greater number of participants reporting a treatment-related $\mathrm{AE}$ in cohort 1 (i.d. administration) was attributable to a higher number of injection-site reactions (for details, see table e-1, links.lww.com/WNL/ A245). In both cohorts, all treatment-emergent injection-site reactions were mild. There were no treatment-related early
Figure 3 Efficacy results in study 1
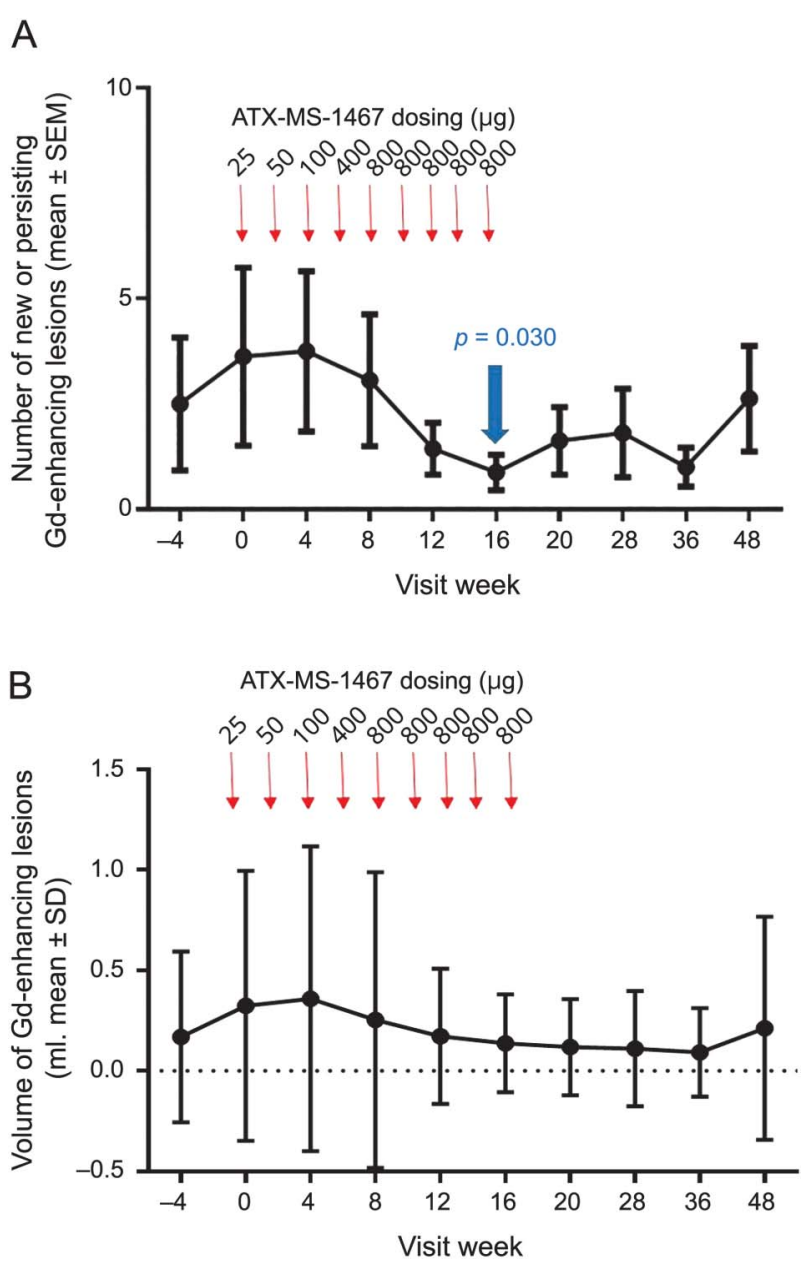

Number $(A)$ and volume $(B)$ of new or persisting gadolinium (Gd)-enhancing T1 lesions over time in the study 1 cohort 1 per-protocol population $(n=16)$. Values are mean \pm SEM $(A)$ and mean $\pm S D(B)$ respectively.

discontinuations. One patient in cohort 1 discontinued the treatment phase because of an $\mathrm{AE}$ (allergic reaction) possibly related to the study medication. There were no severe TEAEs, no treatment-related serious AEs, and no AEs that resulted in dose suspension or withdrawal from the study. There were no reports of drug-related MS relapse in either cohort, nor were there any antibody responses to any ATX-MS-1467 peptides. Treatment-emergent MS relapses were experienced by 2 participants in cohort 1 at weeks 14 and 16, respectively. In cohort 2, 1 participant experienced 2 relapses after treatment at $400 \mu \mathrm{g}$ and at $800 \mu \mathrm{g}$ (weeks 6 and 8) and 2 subjects after treatment at $800 \mu \mathrm{g}$ (weeks 8 and 16). This corresponded to a mean total cohort ARR of 0.15. All participants received steroid rescue therapy and recovered. All relapses were mild or moderate and none was assessed as related to treatment.

In study 2, 15 participants (79\%) experienced a TEAE. All were mild or moderate. Eleven TEAEs (58\%) were identified as treatment-related. The majority were injection-site reactions: erythema in 5 participants (26\%), induration, pain, and 

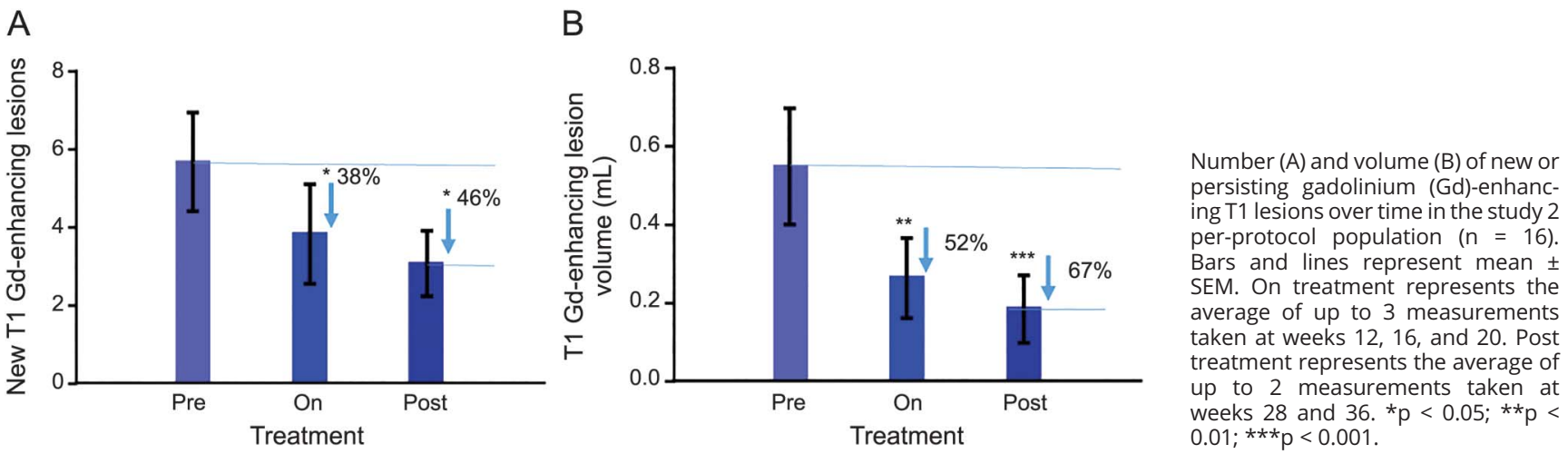

pruritus each in 2 participants (11\%), and hemorrhage in 1 participant (5\%). Two participants (11\%) experienced treatment-related diffuse alopecia. One participant discontinued treatment because of a TEAE of prolonged, moderate diarrhea, which was considered treatment-related.

\section{Discussion}

ATX-MS-1467 is being developed as a potential immunotherapy for MS targeting specifically the autoreactive immune system directed against myelin. This strategy differs from most emerging therapies for MS, which use monoclonal antibodies to target and modify different aspects of the immune system. ${ }^{15,16}$ Although an appealing concept, no peptidebased antigen-specific immunotherapy has yet been successful in MS. The results presented here, from a phase $1 \mathrm{~b}$ and a phase 2a study, further support ATX-MS-1467 as a potentially effective and well-tolerated new therapy.

Dosing and titration regimens are important for antigenspecific immunotherapy. To induce effective tolerance with ATX-MS-1467, a reasonably high dose is considered necessary, as 3 of the $4 \mathrm{MBP}$ peptides in this cocktail display low affinity for major histocompatibility complex binding sites. ${ }^{11}$ Earlier work has demonstrated the critical role of a doseescalation protocol in minimizing $\mathrm{CD}^{+} \mathrm{T}$ cell activation and proliferation during the early stages of immunotherapy, to prevent excessive systemic cytokine release and allow safe administration of the required doses. ${ }^{8}$ The 2 trials described in the present communication provide further information to guide an optimized escalation and treatment scheme for ATXMS-1467 in RMS.

In study 1 , a slower 8 -week titration period was followed by an 8 -week, full-dose treatment period. This corresponded to the regimen in the first in-human study with ATX-MS-1467. ${ }^{11}$ Study 2 used a shorter 4-week titration period and a longer 16week, full-dose treatment period. In both trials, treatment was associated with reductions in the number of T1 GdE lesions, a biomarker for inflammatory disease activity. ${ }^{17,18}$ In study 2, the median number of new/enlarging T2 lesions decreased after week 12 and remained reduced at subsequent visits. Of note, there were no safety and tolerability concerns associated with any treatment regimen.

In study 1 , the reduction in the number of $\mathrm{T} 1 \mathrm{GdE}$ lesions was greater than the reductions in study 2 . However, in the latter trial, the longer full-dose treatment period led to a greater persistence of the effect during off-treatment follow-up. Taken together, these effects on new GdE lesions are comparable to those reported for other MS therapies at similar stages of development. ${ }^{15}$

Although results cannot be directly compared between 2 separate trials, they clearly show that the rate of dose escalation can have a significant effect on efficacy (whereby a longer dose escalation appears to be more favorable). This finding is supported by preclinical data. ${ }^{8}$ In addition, longer treatment with the highest dose appears to offer a longer-lasting effect post treatment.

The difference in outcomes between the 2 cohorts in study 1 is consistent with the i.d. route of administration being optimal for immunotherapy with peptides. It does not exclude the efficacy of the s.c. route, as cohort 2 had fewer GdE lesions at baseline and limited scope for improvement. The consistently low disease activity throughout the course of treatment in cohort 2 may be attributable to a protective effect of ATX-MS1467 , but such conclusions are speculative.

Both studies have limitations, particularly the lack of placebo group or randomization, as well as small cohort sizes. In study 1 , efficacy was evaluated on secondary endpoints. The Paced Auditory Serial Addition Test results should not be overinterpreted, because the score is susceptible to practice effects, particularly during a short trial. ${ }^{19}$ Despite the limitations, these 2 trials, using validated MRI surrogate endpoints ${ }^{20}$ and established outcomes for proof-of-concept and phase 2 efficacy trials in $\mathrm{RMS}^{21}$ support the use of a relatively slow ATX- 
MS-1467 titration procedure combined with a longer fulldose treatment period to achieve effective reduction in $\mathrm{T} 1$ GdE lesions during treatment and a sustained effect post treatment. A further phase $2 \mathrm{~b}$ trial of ATX-MS-1467 in a larger study population is warranted to confirm these findings.

\section{Author contributions}

K.M. contributed to the design and management of study 1 and design of study 2. D.W. designed ATX-MS-1467 and contributed to the design of study 1 and 2. K.B. contributed to the design and management of study 1. J.C. was chief investigator of study 1 . B.S. was an investigator on study 1 . P.S. wrote the manuscript. All authors were involved in the review of the manuscript and approved the final version.

\section{Acknowledgment}

The authors thank the colleagues at Merck KgaA Germany for their contributions to the design and conduct of both studies.

\section{Study funding}

Both studies were funded by Merck KgaA Germany. J.C. acknowledges the UK National Institute for Health Research (NIHR) University College London Hospitals Biomedical Research Centres funding scheme and University College London. D.W. acknowledges the UK NIHR Birmingham Biomedical Research Centres funding scheme.

\section{Disclosure}

J. Chataway has received support from the Efficacy and Mechanism Evaluation Programme and Health Technology Assessment Programme (NIHR); UK Multiple Sclerosis Society and National Multiple Sclerosis Society. In the last 3 years, he has been a local principal investigator for trials in multiple sclerosis funded by Receptos, Novartis, and Biogen Idec, and has received an investigator grant from Novartis outside this work. He has taken part in advisory boards/ consultancy for Roche, Merck KGaA Germany, MedDay, Biogen, and Apitope. K. Martin is employed by Apitope Technology (Bristol) Ltd. K. Barrell is employed by Apitope Technology (Bristol) Ltd. B. Sharrack has received funding from NIHR and the UK MS Society, has been a principal investigator for trials in multiple sclerosis funded by Receptos, Novartis, Biogen, Merck KGaA Germany, Genzyme, Roche, and Teva. P. Stolt is an independent scientific communications consultant. D. Wraith serves as chief scientific officer for Apitope Technology (Bristol) Ltd. and Apitope International $\mathrm{NV}$ on a consultative basis; is on the scientific advisory board for Apitope International NV and has sat on scientific advisory boards for Actelion Pharma and Zealand Pharma; received travel funding from Apitope International NV; is a senior editor for Immunotherapy; holds patents for peptides, tolerisation-inducing composition, FVIII peptides and their use in tolerising haemophiliacs, composition, disease markers, tolerogenic peptides from myelin basic protein, peptide selection method, and improvements relating to influenza vaccine; has consulted for Peptide Therapeutics Ltd., Teva, GSK Bio, Hoffmann-La Roche, Novartis, DTI, and the Food Standards Agency; received research support within the past 3 years from Apitope International NV, UCB Celltech, MRC, the Immune Tolerance Network, and the Wellcome Trust; holds stock and stock options with Apitope International NV; and was an expert witness for Geron. Go to Neurology.org/N for full disclosures.

Received July 31, 2017. Accepted in final form December 8, 2017.

\section{References}

1. Garber K. Immunology: a tolerant approach. Nature 2014;507:418-420.

2. Wingerchuk DM, Carter JL. Multiple sclerosis: current and emerging diseasemodifying therapies and treatment strategies. Mayo Clin Proc 2014;89:225-240.

3. Akdis CA, Akdis M. Mechanisms of allergen-specific immunotherapy and immune tolerance to allergens. World Allergy Organ J 2015;8:17.

4. Bielekova B, Goodwin B, Richert N, et al. Encephalitogenic potential of the myelin basic protein peptide (amino acids 83-99) in multiple sclerosis: results of a phase II clinical trial with an altered peptide ligand. Nat Med 2000;6:1167-1175.

5. Kappos L, Comi G, Panitch H, et al. Induction of a non-encephalitogenic type $2 \mathrm{~T}$ helper-cell autoimmune response in multiple sclerosis after administration of an altered peptide ligand in a placebo-controlled, randomized phase II trial. The Altered Peptide Ligand in Relapsing MS Study Group. Nat Med 2000;6:1176-1182.

6. Freedman MS, Bar-Or A, Oger J, et al. A phase III study evaluating the efficacy and safety of MBP8298 in secondary progressive MS. Neurology 2011;77:1551-1560.

7. Smith CE, Eagar TN, Strominger JL, Miller SD. Differential induction of IgEmediated anaphylaxis after soluble vs. cell-bound tolerogenic peptide therapy of autoimmune encephalomyelitis. Proc Natl Acad Sci USA 2005;102:9595-9600.

8. Burton BR, Britton GJ, Fang H, et al. Sequential transcriptional changes dictate safe and effective antigen-specific immunotherapy. Nat Commun 2014;5:4741.

9. Anderton SM, Viner NJ, Matharu P, Lowrey PA, Wraith DC. Influence of a dominant cryptic epitope on autoimmune T cell tolerance. Nat Immunol 2002;3:175-181.

10. Larché M, Wraith DC. Peptide-based therapeutic vaccines for allergic and autoimmune diseases. Nat Med 2005;11:S69-S76.

11. Streeter HB, Rigden R, Martin KF, Scolding NJ, Wraith DC. Preclinical development and first-in-human study of ATX-MS-1467 for immunotherapy of MS. Neurol Neuroimmunol Neuroinflamm 2015;2:e93.

12. World Medical Association. WMA Declaration of Helsinki: ethical principles for medical research involving human subjects, amended by the 59th WMA General Assembly, Seoul, October 2008 [online]. 2008. Available at: www.wma.net/en/ 30publications/10policies/b3/index.html.pdf. Accessed June 8, 2015.

13. Polman CH, Reingold SC, Banwell B, et al. Diagnostic criteria for multiple sclerosis: 2010 revisions to the McDonald criteria. Ann Neurol 2011;69:292-302.

14. Sormani MP, Miller DH, Comi G, et al. Clinical trials of multiple sclerosis monitored with enhanced MRI: new sample size calculations based on large data sets. J Neurol Neurosurg Psychiatry 2001;70:494-499.

15. Cross AH, Naismith RT. Established and novel disease-modifying treatments in multiple sclerosis. J Intern Med 2014;275:350-363.

16. Northrup L, Christopher MA, Sullivan BP, Berkland C. Combining antigen and immunomodulators: emerging trends in antigen-specific immunotherapy for autoimmunity. Adv Drug Deliv Rev 2016;98:86-98.

17. Sormani MP, Bonzano L, Roccatagliata L, Cutter GR, Mancardi GL, Bruzzi P. Magnetic resonance imaging as a potential surrogate for relapses in multiple sclerosis: a meta-analytic approach. Ann Neurol 2009;65:268-275.

18. Sormani MP, Bruzzi P, Rovaris M, et al. Modelling new enhancing MRI lesion counts in multiple sclerosis. Mult Scler 2001;7:298-304.

19. Tombaugh TN. A comprehensive review of the Paced Auditory Serial Addition Test (PASAT). Arch Clin Neuropsychol 2006;21:53-76.

20. Sormani MP, Bruzzi P. MRI lesions as a surrogate for relapses in multiple sclerosis: a meta-analysis of randomised trials. Lancet Neurol 2013;12:669-676.

21. European Medicines Agency. Guideline on clinical investigation of medicinal products for the treatment of multiple sclerosis [online]. 2015. Available at: http://www. ema.europa.eu/docs/en\%5fGB/document\%5flibrary/Scientific\%5fguideline/2015/ 03/WC500185161.pdf. Accessed April 20, 2017. 


\section{Effects of ATX-MS-1467 immunotherapy over 16 weeks in relapsing multiple sclerosis}

Jeremy Chataway, MA, PhD, FRCP, Keith Martin, PhD, MRPharmS, Kevin Barrell, PhD, Basil Sharrack, MD, PhD, FRCP, Pelle Stolt, PhD, and David C. Wraith, PhD, For the ATX-MS-1467 Study Group

Cite as: Neurology ${ }^{\circledR}$ 2018;90:e955-e962. doi:10.1212/WNL.0000000000005118

\author{
Correspondence \\ Dr. Chataway \\ j.chataway@ucl.ac.uk
}

\section{Trial registration number}

Studies 1 and 2 were registered with the European Clinical Trials Database (number 2009-016710-25) and ClinicalTrials.gov (identifier: NCT01973491), respectively.

\section{Study question}

Under which treatment protocols can ATX-MS-1467 safely and effectively treat relapsing MS (RMS)?

\section{Summary answer}

With slow titration and long full-dose treatment periods, intradermal ATX-MS-1467 can safely reduce the number of lesions in patients with RMS.

\section{What is known and what this paper adds}

A previous small Phase 1 trial provided preliminary evidence that ATX-MS-1467 is tolerable and effective for multiple sclerosis, but the most appropriate titration schemes need to be identified. This study provides Class IV evidence for the safety and efficacy of ATXMS-1467 for RMS under different titration schemes.

\section{Participants and setting}

Study $1\left(\mathrm{n}_{\text {completed }}=39\right)$ involved 9 Russian centers and 2 British centers, and study $2\left(\mathrm{n}_{\text {completed }}=18\right)$ involved 7 Russian centers and a Latvian center. All participants were adults with RMS, defined as at least one relapse in the previous 12 months or 2 in the previous 24, and Expanded Disability Status Scale scores of $\leq 5.5$.

\section{Design, size, and duration}

The study 1 participants received subcutaneous $\left(\mathrm{n}_{\text {completed }}=20\right)$ or intradermal $\left(\mathrm{n}_{\text {completed }}=19\right)$ ATX-MS-1467 administration over an 8-week escalating dose period and an 8-week full-dose period; they were examined off-medication for 32 weeks. The study 2 participants received intradermal ATX-MS-1467 administration over a 4-week escalating dose period and a 16-week fulldose period; they were examined off-medication for 16 weeks.

\section{Primary outcomes}

The primary outcome was the change in the number of visible lesions between pre-study T1 MRI scans and on-treatment T1 MRI scans.

\section{Main results and the role of chance}

The study 1 participants who received intradermal ATX-MS1467 exhibited a reduction from baseline in new and persistent
Figure Number of new or persisting gadolinium-enhanced T1 MRI lesions over time in Study 2 participants

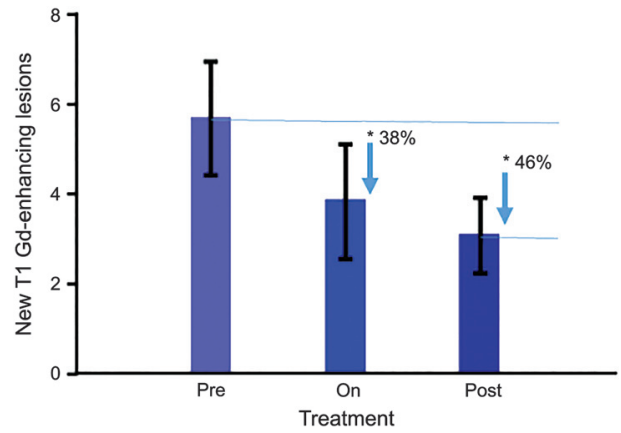

Bars and lines represent means \pm SEMs. "Pre" represents pre-study measurements. "On" represents the average of up to 3 measurements taken in weeks 12,16, and 20. "Post" represents the average of up to 2 measurements taken in weeks 28 and $36 .{ }^{*} p<0.05$.

lesions at week $16(p=0.030)$, although the number had increased by week 48 . The study 2 participants exhibited a reduction from baseline in lesion numbers throughout the study period $(p=0.0143)$.

\section{Harms}

No major adverse events were noted.

\section{Bias, confounding, and other reasons for caution}

The studies had small samples sizes, no placebo groups, and no randomization.

\section{Generalizability to other populations}

Further studies with larger sample sizes are necessary to establish the generalizability of the results.

\section{Study funding/potential competing interests}

The studies were funded by Merck KGaA Darmstadt Germany. Some authors report receiving funding and personal compensation from pharmaceutical companies, holding patents, receiving funding from government agencies, and various other disclosures. Go to Neurology.org/N for full disclosures.

A draft of the short-form article was written by M. Dalefield, a writer with Editage, a division of Cactus Communications. The authors of the full-length article and the journal editors edited and approved the final version. 


\title{
Neurology
}

\section{Effects of ATX-MS-1467 immunotherapy over 16 weeks in relapsing multiple sclerosis}

Jeremy Chataway, Keith Martin, Kevin Barrell, et al.

Neurology 2018;90;e955-e962 Published Online before print February 21, 2018

DOI 10.1212/WNL.0000000000005118

This information is current as of February 21, 2018

\begin{abstract}
Updated Information \&
Services

including high resolution figures, can be found at:

http://n.neurology.org/content/90/11/e955.full.html

References

This article cites 19 articles, 3 of which you can access for free at: http://n.neurology.org/content/90/11/e955.full.html\#\#ref-list-1

Subspecialty Collections

This article, along with others on similar topics, appears in the following collection(s):

All Clinical trials

http://n.neurology.org//cgi/collection/all_clinical_trials

Class III

http://n.neurology.org//cgi/collection/class_iii

Multiple sclerosis

http://n.neurology.org//cgi/collection/multiple_sclerosis

Permissions \& Licensing its entirety can be found online at:

http://n.neurology.org/misc/about.xhtml\#permissions

Reprints

Information about ordering reprints can be found online:

http://n.neurology.org/misc/addir.xhtml\#reprintsus
\end{abstract}

Information about reproducing this article in parts (figures,tables) or in

Neurology ${ }^{\circledR}$ is the official journal of the American Academy of Neurology. Published continuously since 1951, it is now a weekly with 48 issues per year. Copyright @ 2018 American Academy of Neurology. All rights reserved. Print ISSN: 0028-3878. Online ISSN: 1526-632X.

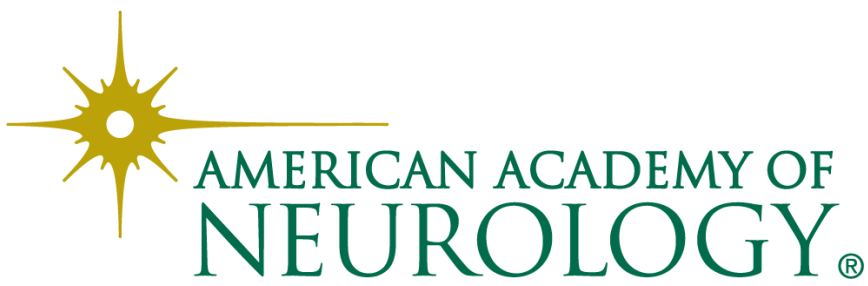

